\title{
Egg specific gravity in improvement of hatchability in laying hens ${ }^{*}$
}

\author{
I. Rozempolska-Rucińska ${ }^{1,3}$, G. Zięba ${ }^{1}$, M. Lukaszewicz², \\ M. Ciechońska ${ }^{1}$, A.Witkowski ${ }^{1}$ and B. Ślaska ${ }^{1}$ \\ ${ }^{1}$ University of Life Sciences in Lublin, Chair for Biological Bases of Animal Production \\ Akademicka 13, 20-950 Lublin, Poland \\ ${ }^{2}$ Institute of Genetics and Animal Breeding, Polish Academy of Sciences \\ Jastrzębiec, 05-552 Wólka Kosowska, Poland
}

(Received 31 August 2010; revised version 3 February 2011; accepted 12 March 2011)

\begin{abstract}
The aim of the study was to assess the suitability of egg specific gravity (ESG) as an accompanying trait in estimation of the birds' breeding value for hatchability. The study involved 3 generations of performance recorded laying hens Rhode Island White (RIW) and Rhode Island Red (RIR). The breeding merit for number of chicks hatched from eggs laid (NCH) was estimated with three different model variants which differed with regard to the information accounted for: 1 . all the standard information plus post factum hatchability record and ESG, 2. no post factum hatchability data on the last generation but ESG present, and 3. no hatchability data on last generation nor ESG. In both lines a high positive correlation between the egg specific gravity and hatchability was observed (0.47 to 0.66). In RIR, when ESG was included in the left-hand-side, a significantly higher concordance of birds' $\mathrm{NCH}$ breeding value ranking between 2. and 1. than between 3 . and 1. were observed.
\end{abstract}

KEY WORDS: laying hens, BLUP, hatchability, egg specific gravity, breeding, (co)variance components

\section{INTRODUCTION}

Improvement and estimation of birds' genetic merits for hatchability is possible mainly based on the relationship matrix. When selecting hens to the breeding flocks we do not have data about their hatching performance. Thus, prediction

\footnotetext{
${ }^{*}$ Supported by the Research Fund for 2008-2011, Project No. N N311 364435

${ }^{3}$ Corresponding author: e-mail: iwona.rucinska@up.lublin.pl
} 
of their breeding value may hardly be reliable and the rank correlation between hens' ranking based on the breeding value predicted on the basis of the preceding generations' hatchability and ranking based on own hatchability record and pedigree is only 0.6 (Rozempolska-Rucińska, 2010). The reliability of the evaluation can be increased either by fitting additional sources of variation (RozempolskaRucińska et al., 2010) or by using correlated egg-laying performance traits, possibly supplemented with other traits estimated early in life of individuals, in the multi-trait approach, or both. Therefore, prior to evaluation of the reproductive performance, it is essential to nominate such hatchability-related traits, which could increase the reliability of the reproduction BLUP proofs.

Besides purely productive traits (egg-laying), hatching success may also be related to the quality of egg shells. The shell is a barrier designed to protect the developing embryo and provide it with an optimal environment. Reduced hatchability and an increased embryonic death rate in chicks incubated from thinshelled eggs have been observed (Bennett, 1992). A proper structure of the shell ensures adequate gas exchange during embryonic development; both egg shell thickness and porosity correlate with hatchability as it was reported, for instance, by Sahan et al. (2003). The quality of the shell is determined by such traits as breaking strength, thickness, pore density, and elasticity which simultaneously correlate with the egg specific gravity (ESG) (Sooncharenying and Edwards, 1989). Hence, since the methods of resistance and thickness measurements are destructive, ESG may serve as an indicator of egg shell quality. Given the specific gravity of fresh eggs, it is possible to assess shell thickness, which in turn is related to porosity and hatchability (Sahan et al., 2003). At the same time, measurement of ESG is one of the cheapest, quickest and non-destructive methods of assessing shell strength (Butcher and Miles, 1991; Cordts et al., 2002). Due to its relation to shell strength, ESG defines the shell internal structure and microstructure, which affect the hatchability success. Eggs of proper specific gravity, ranging from 1.07 to $1.10 \mathrm{~g} / \mathrm{cm}^{3}$, are usually properly shaped, which is particularly important in the case of hatching eggs.

The aim of this study was to evaluate the usefulness of egg specific gravity as an accompanying trait in prediction of the breeding value for hatchability.

\section{MATERIAL AND METHODS}

The study involved three performance recorded generations of 13827 Rhode Island White (RIW) and 6567 Rhode Island Red (RIR) layers. Individual performance recording of the birds included: body weight (BW) in the $18^{\text {th }}$ week of age, sexual maturity - number of days from hatching to laying of the $1^{\text {st }}$ egg 
(AFE), initial laying performance - number of eggs laid during the first 15 weeks of egg-laying (ILN), average weight of eggs laid in the $34^{\text {th }}$ week of hens' life (EW), and average weight of eggs weighted in water (EWW). Using the two latter measurements, ESG was calculated in accordance with the Archimedes 'principle: $\mathrm{ESG}=\mathrm{EW} /(\mathrm{EW}-\mathrm{EWW})$. Hatchability was represented by number of chicks hatched from all the eggs laid during the setting period $(\mathrm{NCH})$, as recommended by Rozempolska-Rucińska et al. (2009). The hatchability traits were recorded exclusively in the reproductive flock. Depending on the breed (RIW or RIR) up to 56 or 42 eggs were laid in the setting season by a hen. Likewise, 0 to 50 and 0 to 40 hatchlings per hen $(\mathrm{NCH})$ were obtained in RIW and RIR, respectively. With so many thresholds phenotypic continuity of $\mathrm{NCH}$ can be supposed. Each year the reproductive hen flock consisted of approximately 530 females and 43 males in the Rhode Island White breed and approximately 290 females and 24 males in the Rhode Island Red one. Table 1 describes the studied traits across the breeds.

Table 1. Numbers, means and standard deviations of studied traits in RIW and RIR

\begin{tabular}{|c|c|c|c|c|c|c|}
\hline \multirow{2}{*}{ Trait } & \multicolumn{3}{|c|}{ Rhode Island White } & \multicolumn{3}{|c|}{ Rhode Island Red } \\
\hline & $\mathrm{n}$ & mean & SD & $\mathrm{n}$ & mean & SD \\
\hline Body weight, $g$ & 13819 & 1582 & 131 & 6567 & 1601 & 143 \\
\hline Age at first egg, days & 13827 & 152 & 9 & 6546 & 146 & 11 \\
\hline Number of eggs during initial laying & 13827 & 87 & 13 & 6546 & 90 & 14 \\
\hline Egg weight, $\mathrm{g}$ & 13171 & 61.7 & 4.5 & 6307 & 61.8 & 4.2 \\
\hline Egg specific gravity, $\mathrm{g} / \mathrm{cm}^{3}$ & 11629 & 1.076 & 0.008 & 6289 & 1.078 & 0.007 \\
\hline Chicks hatched from laid eggs, $\%$ & 1574 & 68.8 & 18.8 & 882 & 78.0 & 16.1 \\
\hline
\end{tabular}

Significance of the effects fitted in the model for estimating the (co)variance components (restricted maximum likelihood - REML) was verified with the multifactor analysis of variance. Components of variance and covariance estimated with models described in Table 2 were subsequently used to predict animals' breeding merits with the BLUP method. For both REML and BLUP runs the DMU package (Madsen and Jensen, 2000) was used.

Table 2. Factors accounted for in the models for particular traits

\begin{tabular}{|c|c|c|c|}
\hline \multirow{2}{*}{ Factor / Type ${ }^{1}$} & \multicolumn{3}{|c|}{ Traits $^{2}$} \\
\hline & BW, AFE, EW, ESG & ILN & $\mathrm{NCH}$ \\
\hline Year of hatch of origin $\mathrm{x}$ hatch number $/ \mathrm{F}$ & $\mathrm{x}$ & $\mathrm{x}$ & \\
\hline Year of reproduction $/ \mathrm{F}$ & & & $\mathrm{x}$ \\
\hline Number of days of initial laying /C & & $\mathrm{x}$ & \\
\hline Number of eggs laid during setting season /C & & & $\mathrm{x}$ \\
\hline Genetic additive effect of the animal /A & $\mathrm{x}$ & $\mathrm{x}$ & $\mathrm{x}$ \\
\hline
\end{tabular}


Four generation pedigrees comprised 21225 RIW and 9845 RIR individuals.

Multi-trait model applied for calculation included traits which themselves were not subjected to direct analyses (BW, AFE, EW, ILN) but facilitated a more accurate estimation of $\mathrm{NCH}$ that was recorded in the final generation (Rozempolska-Rucińska et al., 2008).

The breeding value for $\mathrm{NCH}$ was predicted using three different computation variants which differed in the accompanying sets of traits and in the presence or absence of information about the reproduction success of the last generation, which is referred to as the selected generation. The exact characteristics of the computation variants used is presented in Table 3.

Table 3. Characteristics of the computation variants used in prediction of the birds' breeding value

\begin{tabular}{lccc}
\hline \multirow{2}{*}{ Description of model variants } & \multicolumn{3}{c}{ Variant } \\
\cline { 2 - 4 } Number of performance recorded generations & 3 & 3 & 3. \\
Number of hatchability recorded generations & 3 & 2 & 3 \\
Reproduction record in the selected generation & yes & no & 2 \\
Specific gravity as additional accompanying trait & yes & yes & no \\
Number of hens with recorded hatchability & 882- RIR & 587- RIR & 587- RIR \\
& 1574-RIW & 911-RIW & 911-RIW \\
\hline
\end{tabular}

In the reference variant 1 , , breeding value was predicted using all the chosen performance traits (BW, AFE, EW, ESG, ILN and NCH). The reproduction success of the last, selected generation was also taken into account. In variants 2. and 3., the reproduction success of the last generation was not considered in prediction of the birds' $\mathrm{NCH}$ breeding value. This is a standard procedure in farm practice while predicting the hatchability breeding value and selection of birds for the reproductive flock in the current generation. Variant 2. and 3. differed in whether ESG was included or excluded. Since variant 1 was the most complete, it was regarded the reference variant and the results obtained with variants 2 . and 3. were compared with it. Concordance of individuals' ranking according to their $\mathrm{NCH}$ breeding merits obtained with the use of the different analysis variants was assessed with Spearman's rank correlation.

\section{RESULTS}

Table 4 presents the level of genetic variability of the current traits in the studied hen populations. Depending on the trait, genetic variance ranged from 5 to over $50 \%$ of the total variance. In both breeds, the highest values of the 
heritability coefficient were reported for body weight, sexual maturity and egg weight. The genetic variability of the egg specific gravity was similar in both breeds. However, in the RIR breed, this parameter for the number of hatched chicks had a two-fold lower value.

Table 4. Heritability estimates $\left(\mathrm{h}^{2}\right)$ and their standard errors (se) of studied traits in the RIW and RIR breeds

\begin{tabular}{lccccc}
\hline \multirow{2}{*}{ Trait $^{1}$} & \multicolumn{2}{c}{ Rhode Island White } & & \multicolumn{2}{c}{ Rhode Island Red } \\
\cline { 2 - 3 } \cline { 5 - 6 } \cline { 5 - 6 } & $\mathrm{h}^{2}$ & SE & & $\mathrm{h}^{2}$ & SE \\
\hline BW & 0.56 & 0.02 & & 0.55 & 0.03 \\
AFE & 0.41 & 0.02 & & 0.28 & 0.03 \\
ILN & 0.07 & 0.01 & & 0.05 & 0.01 \\
EW & 0.40 & 0.02 & & 0.44 & 0.03 \\
ESG & 0.18 & 0.02 & & 0.21 & 0.02 \\
NCH & 0.22 & 0.05 & & 0.10 & 0.04
\end{tabular}

${ }^{\mathrm{T}}$ explanation - see Table 2

The correlations between the egg specific gravity and the other accompanying traits are shown in Table 5. It is noteworthy that there is a high negative correlation between ESG and EW as well as positive correlations with hatchability; this is particularly distinct in RIR, where the genetic correlation was 0.66 and of a small standard error (0.06).

No significant correlations with the ESG were found for the other traits.

Table 5. Genetic correlations $\left(\mathrm{r}_{\mathrm{G}}\right.$ ) between specific gravity and other traits in the RIW and RIR lines (SE - standard error)

\begin{tabular}{|c|c|c|c|c|}
\hline \multirow{2}{*}{ Traits } & \multicolumn{2}{|c|}{ Rhode Island White } & \multicolumn{2}{|c|}{ Rhode Island Red } \\
\hline & $\mathrm{r}_{\mathrm{G}}$ & $\mathrm{SE}$ & $\mathrm{r}_{G}$ & $\mathrm{SE}$ \\
\hline Body weight & -0.18 & 0.08 & -0.08 & 0.07 \\
\hline Sexual maturity & 0.14 & 0.07 & -0.02 & 0.05 \\
\hline Initial laying number of eggs & -0.03 & 0.05 & 0.12 & 0.05 \\
\hline Egg weight & -0.36 & 0.07 & -0.34 & 0.06 \\
\hline Hatched chicks & 0.47 & 0.06 & 0.66 & 0.06 \\
\hline
\end{tabular}

The supplementary information about ESG did not affect the BLUP predictions of hatchability in a way that would lead to changes in birds' ranking in RIW (Table 6).

Table 6. Rank correlations between the NCH breeding merits obtained with the use of computation model variant 1 . and those obtained with the other variants

\begin{tabular}{lll}
\hline Model variant & RIW & RIR \\
\hline 2. & 0.76 & 0.84 \\
3. & 0.75 & 0.51 \\
\hline
\end{tabular}

The rank correlations between variant 1 . and variants 2 . and 3 . were similar in RIW. Yet, thanks to the application of the multi-trait model with ESG, 
a significant increase in the accuracy of $\mathrm{NCH}$ breeding values was obtained in RIR; the concordance of birds' ranking between the reference 1. and predicted with 2. or 3 . NCH breeding values increased from 0.51 (3.) to 0.84 (2.).

\section{DISCUSSION}

Given the fact that information provided by other traits recorded in birds enhanced the reliability of BLUPs for the hatchability traits (RozempolskaRucińska et al., 2008), these traits (characterized in Tables 1 and 4) were routinely included in each of the computation variants. The genetic variability found in the population was in the range described by other authors (Poggenpoel et al., 1996; Szwaczkowski, 1999; Zięba et al., 2003; Mielenz et al., 2004; RozempolskaRucińska et al., 2007). Differences in the value of parameters are related to the pedigree structure of the studied populations, mathematical models applied or prediction methods.

The genetic additive variability of ESG constituted approximately $20 \%$ of the total variability of the trait. This value was lower than that obtained by Sabri et al. (1999), where heritability ranged from 0.23 to 0.45 , depending on the egg-laying period. The investigations conducted by other authors involved shell resistance (Nirasawa et al., 1998; Besbes and Gibson, 1999), correlated with ESG. In that case, the value of that parameter was also slightly higher and ranged from 0.23 to 0.38 .

The analysis of the present results demonstrated a two-fold higher coefficient of NCH heritability in the RIW line, compared with RIR. The time span of selection and its intensity have been similar in both lines; thus, it may not explain the differences between the values of the heritability coefficient.

The relatively lower additive variability of the number of hatched chicks in the RIR line may impede birds' genetic progress. It was found that even in the RIW breed, where the heritability coefficient was higher, the concordance of ranking between the reference breeding merits and predicted breeding merits for hatchability traits was approximately 0.60 (Rozempolska-Rucińska, 2010). The analysis of the results obtained indicates that recording of the egg specific gravity can be recommended for improvement of the hatchability traits. Such selection is justified in the case of highly correlated traits, such as the egg specific gravity and the number of chicks hatched from laid eggs (Table 5). These traits were found to be very highly, positively correlated, particularly in the RIR line. These are favourable circumstances as determination of the egg specific gravity is possible prior to selection of birds for the reproductive flock. On the other hand, the egg specific gravity is a trait that broadly characterises the quality of eggs 
that are used in hatching. The egg specific gravity indirectly defines the shape of an egg, resistance and structure of the shell, i.e. traits that are essential for a normal hatching process. In selection for the genetic improvement of this trait, we should reduce the percentage of eggs which are disqualified from hatching. Thus, there is a possibility that the hatchability of laid eggs increases. Nowadays, hatching from laid eggs ranges from 68 to $78 \%$ (Table 1); since the percentage of chicks hatched from set eggs is by 10 higher (unpublished data), this difference results from disqualification of eggs designated for hatching, and which is most frequently caused by defects of the shell structure.

On the other hand, egg specific gravity appeared to be negatively correlated with egg weight (Table 5). The higher the egg weight, the thinner the shells, probably due to an insufficient supply of calcium required for shell formation. Simultaneously, the mass gain of the whole egg is quicker than the gain of the shell weight; therefore, bigger-mass eggs may have a smaller proportion of the shell. Negative correlations between the egg specific gravity and egg weight may be a desirable phenomenon. If selection aims at improvement of specific gravity, one can control egg weight as a correlated response so as to prevent production of too big eggs which negatively affects the hatching success. Negative correlations between hatchability traits and egg weight have commonly been reported (e.g., Rozempolska-Rucińska et al., 2007). Since too small eggs cannot guarantee high hatching success, one should compromise and take into account proper emphasis on both these selection criteria (Nirasawa et al., 1998).

The results presented in Table 6 confirm the advisability of using the egg specific gravity in improvement of hatchability traits. In the RIR breed, a considerable increase of concordance of NCH birds' predicted ranking with the reference ranking has been stated, when the model included information about the egg specific gravity. The ranking concordance between the real and predicted breeding merits increased from approximately 0.5 to over 0.8 (variant 2.). No such dependencies have been reported for the RIW line. This may possibly be explained by lower genetic correlation between the particular traits, which may be a result of somewhat different selection pressures. On the other hand, the rank correlations between the reference breeding values and those determined by calculation variant 3 . were markedly higher in RIW than in RIR. This may be interpreted by the fact that both traits in the RIW breed - the egg specific gravity and the number of hatched chicks - are genetically determined to a similar extent $(0.18$ and 0.22$)$. If trait heritabilities are similar, estimation of one trait on the basis of assessment of the other does not yield the expected result; therefore, indirect selection is groundless. 


\section{CONCLUSIONS}

The results of the study indicate that measurement of egg specific gravity and use of this trait as an accompanying trait in prediction of breeding value for hatchability may result in increased reliability of such prediction in layers, although its usefulness may be population dependent.

\section{REFERENCES}

Bennett C.D., 1992. The influence of shell thickness on hatchability in commercial broiler breeder flocks. J. Appl. Poultry Res. 1, 61-65

Besbes B., Gibson J.P., 1999. Genetic variation of egg production traits in purebred and crossbred laying hens. Anim. Sci. 68, 433-439

Butcher G.D., Miles R.D., 1991. Egg specific gravity - designing a monitoring program. http://edis. ifas.ufl.edu/vm044 (accessed 03.02.2011)

Cordts C., Schmutz M., Preisinger R., 2002. New alternatives for improving egg shell stability through breeding. Lohmann Inform. 26, 1-4

Madsen P., Jensen J., 2000. A user's guide to DMU - a package for analyzing multivariate mixed models. Version 6, release 4. Danish Institute of Agricultural Sciences

Mielenz N., Nurgiartiningsih V.M.A., Schmutz M., Preisinger R., Schüler L., 2004. Estimation of genetic parameters based on single and group data from several generations in laying hens. In: Proceedings of XXII World Poultry Congress, Istanbul (Turkey), 132 (G3), pp. 8-13

Nirasawa K., Takahashi H., Takeda H., Furukawa T., Takeda T., Nagamine Y., 1998. Restricted maximum likelihood estimates of genetic parameters and genetic trends of chickens divergently selected for eggshell strength. J. Anim. Breed. Genet. 115, 375-381

Poggenpoel D.G., Ferreira G.F., Hayes J.P., Preez J.D., Du Preez J.J., 1996. Response to long-term selection for egg production in laying hens. Brit. Poultry Sci. 37, 743-756

Rozempolska-Rucińska I., 2010. Reliability of breeding value estimation of laying hens for hatching characteristics. Annal. Anim. Sci. 10, 49-55

Rozempolska-Rucińska I., Twardowska M., Zięba G., Łukaszewicz M., Witkowski A., 2008. Our knowledge of hens' reproductive breeding value is limited upon selecting birds for flock reproduction. Anim. Sci. Pap. Rep. 26, 79-84

Rozempolska-Rucińska I., Zięba G., Łukaszewicz M., 2009. Hatchability traits as selection criteria in breeding of laying hens. Arch. Geflügelk. 73, 263-267

Rozempolska-Rucińska I., Zięba G., Łukaszewicz M., 2010. Service cock only indirectly affects hatchability in laying hens. Anim. Sci. Pap. Rep. 28, 153-159

Rozempolska-Rucińska I., Zięba G., Twardowska M., Łukaszewicz M., Witkowski A., 2007. Relationships between hatchability and routine selection criterion in laying hens. In: Proceedings of XIX International Poultry Science PB WPSA, Olsztyn (Poland), pp. 45-48

Sabri H.M., Wilson H.R., Harms R.H., Wilcox C.J., 1999. Genetic parameters for egg and related characteristics of White leghorn hens in a subtropical environment. Genet. Mol. Biol. 22, 183186

Sahan U., Altan Ö., Ipek A., Yilmaz B.,2003. Effects of some egg characteristics on the mass loss and hatchability of ostrich (Struthio camelus) eggs. Brit. Poultry Sci. 44, 380-385

Sooncharenying S., Edwards H.M., 1989. Modelling the relationships of egg weight, specific gravity, shell calcium and shell thickness. Brit. Poultry Sci. 30, 623-631 
Szwaczkowski T., 1999. Additive and additive-by-additive genetic variability of productive traits in laying hens. J. Anim. Feed Sci. 8, 191-201

Zięba G., Łukaszewicz M., Twardowska M., Witkowski A., 2003. Genetic trends of laying merit in paternal and maternal strains of hens. Anim. Sci. Pap. Rep. 21, 241-249 